\title{
Periodontitis and polycystic ovary syndrome: An unveiling review
}

\author{
Preetika Bansal ${ }^{1}$, Sukhmeet Kaur ${ }^{2}$, Chandni Mittal ${ }^{3}$ \\ ${ }^{1}$ Professor, ${ }^{2}$ Assistant Professor, ${ }^{3}$ BDS Lecturer, Dept. of Periodontics, Dasmesh Institute of Research \& Dental Sciences, \\ Faridkot, Punjab, India
}

*Corresponding Author:

Email: dr_preetika_11@yahoo.co.in

\begin{abstract}
Polycystic ovary syndrome (PCOS) is a complex hormonal disorder affecting $\approx 6 \%$ of women of reproductive age in which the ovaries contain many cystic follicles and associated with overproduction of sex hormones. Increased levels of these sex hormones are associated with increased gingival inflammation. PCOS is one of the main causes of infertility and also shows an interacting link with oral health. PCOS may alter the composition of the oral micro flora through various mechanisms. The sex hormones play a role in the maintenance of bone mass and directly or indirectly influence periodontal cells. Thus the aim of this review article is to find out the association between periodontal diseases and polycystic ovary syndrome.
\end{abstract}

Keywords: PCOS, Sex hormones, Gingival inflammation, Periodontal diseases, Oral micro flora.

\section{Introduction}

Polycystic ovary syndrome (PCOS), or SteinLeventhal syndrome, is a genetically complex endocrine disorder of uncertain etiology affecting about $6.5 \%$ to $8 \%$ of women in reproductive ages and associated with metabolic dysfunction. ${ }^{1,2}$ Polycystic ovary syndrome (PCOS) is a common cause of anovulatory infertility. ${ }^{3,4}$ Potential metabolic risk factors associated with this syndrome are: Type 1 diabetes mellitus, endothelial dysfunction, visceral obesity $^{5}$ and chronic low grade inflammation ${ }^{6}$ places these patients at a greater risk for development of symptomatic atherosclerotic cardiovascular diseases. Therefore, PCOS may be considered as a suitable model for studying the complex interaction among these cardiovascular risk factors, especially chronic inflammation. Periodontitis is a chronic infectious multifactorial disease which leads to alveolar bone loss and cause local and systemic elevations of proinflammatory cytokines, such as tumor necrosis factor-a (TNF-a), interleukin-6 (IL-6) and interleukin17 (IL-17). ${ }^{7}$ It is already confirmed through the various evidences that there is an association between periodontitis and systemic disorders, including metabolic syndrome, diabetes, and cardiovascular disease (CVD). ${ }^{8-10}$ Because of the fact that both periodontal disease and metabolic syndrome are associated with systemic inflammation and insulin resistance, these two disorders may be shared a common pathophysiologic pathway. ${ }^{8}$ PCOS appears to be linked with increased oxidative stress and systemic inflammation because females with PCOS have increased markers of lipid peroxidation, C-reactive protein (CRP), inflammatory cytokines. ${ }^{11,12}$ Still there is limited information regarding periodontal health, the oral microbiota, in relation with systemic inflammatory conditions caused by PCOS. The aim of this paper was to review the literature focusing on the oral aspects of PCOS.

\section{PCOS and gingival inflammation}

In response to the bacterial infection, host derived inflammatory mediators (cytokines) and proteolytic enzymes (MMPs) are triggered and activated. ${ }^{13}$ PCOS is characterized by chronic low-grade inflammation ${ }^{14}$ and a prolonged low-grade inflammatory state can be caused by chronic infections such as gingivitis. It was observed that gingivitis is frequently present in patients with PCOS. Gingivitis is a reversible condition associated with inflammation caused by the microbial biofilm accumulating on the tooth surfaces. If left untreated, gingivitis leads to periodontitis in some individuals. Regarding the association of PCOS with gingival inflammation, it was recently demonstrated that they appear to act synergistically on the production of proinflammatory cytokines IL-6, IL-17 and TNF$\alpha^{15,16}$ Thus, PCOS may have an impact on gingival inflammation or viceversa (Ozcaka $O$ et al 2012)..$^{15} \mathrm{IL}$ 17 levels are altered in non-obese females with PCOS and may influence gingival inflammation (Ozcaka $\mathbf{O}$ et al 2013). ${ }^{16}$ Matrix metalloproteinase's (MMP's) play an important role in physiological and pathological processes including inflammatory response, cardiovascular diseases, and ovarian dysfunction. In a study done by Akcalı A et al (2015) ${ }^{17}$ it was concluded that increased levels of MMP-8 and MMP-8/TIMP-1 ratio in saliva and serum in women with PCOS and potentiated by gingival inflammation.

\section{PCOS and oral microflora}

In vivo and in vitro investigations examined bacterial cultures during periods of change in hormonal levels and observed the effect of female sex steroids on the composition of the subgingival biofilm and showed positive link. Pregnancy, puberty, menstruation and 
menopause are four physiological conditions which are associated with fluctuations in endogenous hormonal levels and offer a means to analyze the changes occur in the oral microbial community. Oral contraceptive use and hormone replacement therapy alters the hormonal levels and also used to examine the effect of exogenous hormonal fluctuations on oral microbial profiles. ${ }^{18}$ Ozcaka $O$ et al. (2013) ${ }^{16}$ reported increased salivary levels of the selected pathogens (Porphyromonas gingivalis, Fusobacterium nucleatum, Streptococcus oralis, and Tannerella forsythia) and serum-antibody response in women with PCOS. Akali et al. (2014) ${ }^{19}$ have compared the levels of putative periodontal pathogens in saliva and their antibody response in serum in PCOS patients with healthy subjects and found that the women with PCOS, salivary Porphyromonas gingivalis, Fusobacterium nucleatum, Streptococcus oralis and Tannerella forsythia levels were higher than healthy women. The presence of PCOS also enhanced $P$. gingivalis, Prevotella intermedia and $S$. oralis serum antibody levels. Gingival inflammation correlated positively with levels of these mentioned microfloras in saliva in PCOS patients. PCOS may quantitatively affect the composition of oral microflora and exerting a confounding role in resultant gingival inflammation and periodontal health. The most concordant effect seems to be exerted on $P$. gingivalis. $^{19}$

\section{PCOS and periodontitis}

As we discussed earlier that PCOS may have an impact on gingival inflammation or viceversa (Ozcaka O et al 2012). ${ }^{15}$ If not treated lead to periodontitis. CRP is a serologic marker of systemic inflammation that increased the risk for various systemic diseases. ${ }^{20} \mathrm{CRP}$ levels increased in periodontitis so CRP might be a possible mediator of the association between periodontitis and these systemic conditions. ${ }^{21}$ Patients with periodontitis also have a higher concentration of pro-inflammatory cytokines such as tumor necrosis factor (TNF)-a and interleukin (IL)-1 in serum and/or gingival crevicular fluid. ${ }^{22,23}$ Chronic secretion of these proinflammatory cytokines in periodontal infection may lead to insulin resistance. ${ }^{24}$ This pathognomonic state of chronic systemic inflammation and insulin resistance in both periodontitis and PCOS can be hypothesized as a converging channel associating the disorders. In a study done by Porwal S et al in (2014) ${ }^{25}$ observed influence of systemic inflammation (hsCRP) on periodontal parameters, which showed a strong association of hsCRP with periodontal inflammation and breakdown, suggesting that increased systemic inflammation due to PCOS may influence the periodontal condition. In addition, sex hormones (androgens, estrogens and progestins) play an important role in the maintenance of bone mass, and directly and indirectly influence periodontal cells. ${ }^{19}$

\section{Prevalence of periodontal disease in PCOS}

Porwal S et al (2014) ${ }^{25}$ did a cross-sectional, single-masked study and resulted that the women with newly diagnosed PCOS may have increased prevalence for periodontitis, with higher measures of periodontal inflammation and breakdown than those on medical treatment for PCOS and systemically healthy females. Rahiminejad et al (2015) ${ }^{26}$ conducted a cross-sectional study to find out the prevalence of periodontitis in females with PCOS and found that prevalence of periodontal disease parameters were higher in nonobese women with PCOS comparing to systematically healthy controls. Another study done by Nair SD et al (2017) ${ }^{27}$ observed higher prevalence of periodontal disease parameters in the PCOS group as compared to the women without PCOS.

\section{Conclusion}

The studies reviewed in this article suggest that patients with PCOS have altered levels of some markers of inflammation, which reflects a state of chronic low grade gingival inflammation and PCOS shows a positive and significant association with the clinical and molecular parameters of periodontal diseases. However, long term significance of these findings yet to be confirmed.

\section{References}

1. Stein IF, Levental ML (1935). Amenorrhea associated with bi-lateral polycystic ovaries. Am J Obstet Gynecol; 29: 181-91.

2. Norman RJ, Dewailly D, Legro RS, Hickey TE (2007). Polycystic ovary syndrome. Lancet; 370:685-97.

3. Hull MG (1987). Epidemiology of infertility and polycystic ovarian disease: Endocrinological and demographic studies. Gynecol Endocrinol;1:235-45.

4. Azziz R (2003). The evaluation and management of hirsutism. Obstet Gynecol;101:995-1007.

5. Lim SS, Norman RJ, Davies MJ, Moran LJ (2013). The effect of obesity on polycystic ovary syndrome: a systematic review and meta-analysis. Obes Rev;14:95109.

6. Kelly CC, Lyall H, Petrie JR, Gould GW, Connell JM, Sattar N (2001). Low grade chronic inflammation in women with polycystic ovarian syndrome. J Clin Endocrinol Metab;86:2453-5.

7. Ebejer, K. and Calleja-Agius J (2013). The role of cytokines in polycystic ovarian syndrome. Gynecol Endocrinol; 29:536-40.

8. D'Aiuto F, Sabbah W, Netuveli G, Donos N, Hingorani AD, Deanfield J, Tsakos G (2008). Association of the metabolic syndrome with severe periodontitis in a large U.S. population-based survey. J Clin Endocrinol Metab;93:3989-94.

9. Kinane D, Bouchard P (2008). Group E of European Workshop on Periodontology. Periodontal diseases and health: Consensus report of the Sixth European Workshop on Periodontology. J Clin Periodontol;35(Suppl. 8):333-7.

10. Bullon P, Morillo JM, Ramirez-Tortosa MC, Quiles JL, Newman HN, Battino M (2009). Metabolic syndrome and periodontitis: Is oxidative stress a common link? J DentRes;88:503-18. 
11. Orio F Jr, Palomba S, Cascella T, Di Biase S, Manguso F, Tauchmanova L, et al (2005). The increase of leukocytes as a new putative marker of low-grade chronic inflammation and early cardiovascular risk in polycystic ovary syndrome. J Clin Endocrinol Metab;90:2-5.

12. Knebel B, Janssen OE, Hahn S, Jacob S, Gleich J, Kotzka J, Muller-Wieland D (2008). Increased low grade inflammatory serum markers in patients with polycystic ovary syndrome (PCOS) and their relationship to PPARgamma gene variants. Exp Clin Endocrinol Diabetes;116:481-6.

13. Salminen A, Gursoy UK, Paju S, Hyvarinen K, Mantyla P, Buhlin K, et al (2014). Salivary biomarkers of bacterial burden, inflammatory response, and tissue destruction in periodontitis. J Clin Periodontol; 41:442-50.

14. Repaci A, Gambineri A, Pasquali R (2011). The role of low-grade inflammation in the polycystic ovary syndrome. Mol Cell Endocrinol;335:30-41.

15. Ozcaka O, Ceyhan BO, Akcali A, Bicakci N, Lappin DF, Buduneli N (2012). Is there an interaction between polycystic ovary syndrome and gingival inflammation? J Periodontol;83:1529-37.

16. Ozçaka O, Buduneli N, Ceyhan BO, Akcali A, Hannah V, Nile C, et al (2013). IL-17 involved in the interaction between polycystic ovary syndrome and gingival inflammation? J Periodontol; 84:1827-37.

17. Akcalı A, Bostanci N, Ozçaka O, Ozturk-Ceyhan B, Gumuş P, Tervahartiala T, Husu H, et al (2015). Elevated matrix metalloproteinase 8 in saliva and serum in polycystic ovary syndrome and association with gingival inflammation. Innate Immune; 21(6):619-25.

18. Kumar PS (2013). Sex and the subgingival microbiome: do female sex steroids affect periodontal bacteria? Periodontol 2000;61(1):103-24.

19. Akcalı A, Bostanci N, Ozçaka O, Ozturk-Ceyhan B, Gumuş P, Buduneli N, et al (2014). Association between polycystic ovary syndrome, oral microbiota and systemic antibody responses. PLOS One Sep 18;9(9):e108074.

20. Slade GD, Offenbacher S, Beck JD, Heiss G, Pankow JS (2000). Acute-phase inflammatory response to periodontal disease in the US population. J Dent Res;79:49-57.

21. Pitiphat W, Savetsilp W, Wara-Aswapati N (2008). Creactive protein associated with periodontitis in a Thai population. J Clin Periodontol; 35:120-5.

22. Loos BG, Craandijk J, Hoek FJ, Wertheim-van Dillen PM, van der Velden U (2000). Elevation of systemic markers related to cardiovascular diseases in the peripheral blood of periodontitis patients. $\mathrm{J}$ Periodontol;71:1528-34.

23. Engebretson S, Chertog R, Nichols A, Hey-Hadavi J, Celenti R, Grbic J (2007). Plasma levels of tumour necrosis factor-alpha in patients with chronic periodontitis and type 2 diabetes. J Clin Periodontol;34:18-24.

24. Reaven GM (2011). Insulin resistance: The link between obesity and cardiovascular disease. Med Clin North Am;95:875-92.

25. Porwal S, Tewar S, Sharma RK, Singhal SR, Narula SC (2014). Periodontal status and high-sensitivity C-reactive protein levels in polycystic ovary syndrome with and without medical treatment. J Periodontol;85:1380-9.

26. Rahiminejad ME, Moaddab A, Zaryoun H, Rabiee S, Moaddab A, Khodadoustan AK (2015). Comparison of prevalence of periodontal disease in women with polycystic ovary syndrome and healthy controls. Dent Res J;12:507-12.
27. Nair SD, Varma S, Suragimath G, Zope S, Kale V, Abbayya K (2017). Prevalence of periodontal disease in women with polycystic ovary syndrome- A comparative descriptive study. J Evolution Med Dent Sci;6(65):47336. 